\title{
Coordination Dynamics of Multi-Agent Human Interaction
}

\author{
Shannon Proksch ${ }^{1, *}$, Majerle Reeves ${ }^{2}$, Michael Spivey ${ }^{1}$, and Ramesh Balasubramaniam ${ }^{1}$ \\ ${ }^{1}$ University of California-Merced, Cognitive and Information Sciences, Merced, USA \\ ${ }^{2}$ University of California-Merced, Applied Mathematics, Merced, USA \\ *sproksch@ucmerced.edu
}

\begin{abstract}
Humans interact with other humans at a variety of timescales and in a variety of social contexts. We exhibit patterns of coordination that may differ depending on whether we are genuinely interacting as part of a coordinated group of individuals vs merely co-existing within the same physical space. Moreover, the local coordination dynamics of an interacting pair of individuals in an otherwise non-interacting group may spread, propagating change in the global coordination dynamics and interaction of an entire crowd. Dynamical systems analyses, such as Recurrence Quantification Analysis (RQA), can shed light on the underlying coordination dynamics of multi-agent human interaction. We used RQA to examine the coordination dynamics of a performance of "Welcome to the Imagination World", composed for wind orchestra. This performance enacts a real-life simulation of the transition from uncoordinated, non-interacting individuals to a coordinated, interacting multi-agent group. Unlike previous studies of social interaction in musical performance which rely on different aspects of video and/or acoustic data recorded from each individual, this project analyzes group-level coordination patterns solely from the group-level acoustic data of an audio recording of the performance. Recurrence and stability measures extracted from the audio recording increased when musicians coordinated as an interacting group. Variability in these measures also increased, indicating that the interacting ensemble of musicians were able to explore a greater variety of behavior than when they performed as non-interacting individuals. As an orchestrated (non-emergent) example of coordination, we believe these analyses provide an approximate upper bound for recurrence patterns that may be observable before and after truly emergent coordination.
\end{abstract}

\section{Introduction}

Science has looked to art for inspiration in explaining human cognition. Music, in particular, has aided scientists exploring human engagement with the world, from emotional experience ${ }^{1-3}$ to social interaction ${ }^{4-8}$. Music provides an ideal model system of human social interaction-balancing ecological validity of the interaction and environment with experimental control $^{4}$. Humans interact with other humans in a variety of timescales and social contexts. We exhibit patterns of coordination that may differ whether we are genuinely interacting as part of a coordinated group vs merely co-existing within the same physical space. Moreover, the local coordination dynamics of an interacting pair in an otherwise non-interacting group may spread, propagating a change in the global coordination dynamics and interaction of an entire crowd. Consider the initiation of the slow clap by one, then two, then four people, before breaking into full audience applause or the first musician in a flash mob initiating a flow of musicians and audience members engaging in shared music making. Studying the patterns which arise from the emergence of interaction in a group of people can shed light on the emergent underlying coordination dynamics of multi-agent human interaction.

Transitions from disorder to order are exhibited by a variety of animals ranging from locusts marching ${ }^{9}$ to birds flocking ${ }^{10}$ to humans clapping ${ }^{11}$. The patterns and conditions for this emergent coordination between individuals has been a subject of laboratory study for decades. Spontaneous, or emergent, patterns of entrainment are measured between individuals by analyzing video and motion capture from interacting dyads swinging pendulums ${ }^{12}$ or rocking in rocking chairs ${ }^{13}$. Recent work has evaluated motor coordination dynamics of naturalistic interactions such as interactive problem solving ${ }^{14,15}$, naturalistic conversation between individuals ${ }^{16,17}$, speed-dating partners ${ }^{18}$, and motor and acoustic coordination of performing musicians in duets ${ }^{5,7}$ and larger ensembles ${ }^{6,8}$. In these interactions, behavioral output of each interacting individual was measured and analyzed for meaningful correlations between individuals. An investigation of emergent synchrony in audience applause explicitly measured acoustic output of the group ${ }^{11}$. However, the motor behavioral patterns were still measured from the local behavior of individual audience members in order to evaluate correlation with the global signal of the audience. In each of these studies, it has been possible to obtain clear measurements of individual behavior to examine the emergent coordination dynamics of multi-agent interaction and social self-organization. However, if that multi-agent group has indeed self-organized into a complex system, then the interdependence between the agents' functions should make it possible to detect that coordination 
from almost any time series emitted from that system, using state-space reconstruction ${ }^{19,20}$. Thus, if obtaining movement or acoustic measurements at the individual and local level is not feasible or practical with group behaviors in the wild, then global-level measurements should suffice. One of the simplest global measures to employ is an acoustic recording taken from a well-placed microphone.

The aim of this paper is to show that it is possible to describe coordination patterns of multi-agent interaction by analyzing a time series extracted solely from group-level data. Rather than analyzing the individual-level behavior of interacting agents, we used nonlinear methods from the dynamical systems framework on group-level acoustic data. We analyzed global patterns of coordination in a musical performance of "Welcome to the Imagination World" 21 . This performance enacts a physical simulation of an orchestrated (non-emergent) transition from uncoordinated to coordinated interaction. We used Recurrence Quantification Analysis (which relies on state-space reconstruction) to investigate patterns of coordination from the audio signal of a performance of this work ${ }^{22}$. We believe recurrence measures of this orchestrated musical performance provide a possible upper bound for recurrence patterns that may be observable before and after spontaneous emergent coordination. Moreover, analysis of the transition itself from uncoordinated to coordinated behavior may provide insight into the trade-off between the playful enjoyment of novelty and the rigor of predictive success ${ }^{23}$. Finally, we discuss applications to other examples of real-world multi-agent human interactions, such as multi-agent interaction at sporting events or of individuals coordinating in a protest.

\section{Human Behavior as a Complex Dynamical System}

\section{Principles of Complex Dynamical Systems}

A canonical example of a simple dynamical system is the pendulum clock. A pendulum is a mechanical device-e.g. a fixed weight on a string-which oscillates isochronously around a central point, meaning that swings in both directions take equal amounts of time. The consistent rate of oscillation made the pendulum clock an ideal time-keeper following its invention in the seventeenth century by Cristian Huygens. The pendulum clock and the metronome are examples of simple dynamical systems. The state (location) of the pendulum at any given time is determined by the trajectory of the pendulum over historical time. The oscillatory behavior of a pendulum can be explained by a system of differential equations.

What is relevant here is the behavior that emerges among two or more pendulums placed on a shared surface. Huygens observed that two pendulums hanging from a single beam will spontaneously_or emergently-synchronize their behavior, swinging simultaneously in anti-phase with one another. Multiple metronomes placed atop a platform balanced on two cylinders will also demonstrate emergent synchronized behavior ${ }^{24,25}$. A metronome is a special type of pendulum, which clicks at isochronous intervals to aid time-keeping for musicians. Metronomes feature a fixed weight at the base of a rod, in addition to a moveable weight which slides along the top of the rod to adjust the speed of the metronome oscillations, and thus the speed (tempo) of the audible metronome clicks. If set at the same tempo, the oscillating pendulum and audible clicks of multiple metronomes will begin to synchronize both in-phase and anti-phase with one another ${ }^{25}$. What begins as multiple individual metronome clicks will transition to clicks occurring simultaneously, as globally isochronous acoustic events. For both the pendulum clocks and the clicking metronomes, the local behavior of each individual pendulum is coupled to the behavior of the surrounding pendulums due to their behavior within a shared context — in this case a physical connection via a single beam or a single platform. The local behavior of each individual metronome or pendulum (each oscillating at approximately the same frequency but different phase) eventually self-organizes into emergent global patterns of synchronized behavior.

We observe similar patterns of behavioral synchronization in multi-agent human interaction. The shared context for multi-agent human behavior need not be a physical connection like the metronomes' shared platform. Rather, the shared context mediating emergent global patterns of human behavior is the interaction itself. When local behavior of individual human agents becomes coupled to the behavior of surrounding agents - via the shared context of interaction-the agents self-organize to exhibit emergent global patterns of coordinated behavior. We can detect this emergent coordination of multi-agent human groups from their acoustic behavior over time, just as we can detect the emergent coordination of metronomes from their acoustic output over time.

\section{Recurrence Quantification Analysis}

Multi-agent human behavior, such as in crowds or musical ensembles, can be considered as complex dynamical systems, where complex global patterns of behavior emerge as a result of the self-organization of individual agents over time acting according to simple local rules. The behavior of such a dynamical system can be visualized in recurrence plots. These recurrence plots display the system's trajectory through a phase-space, depicting when that trajectory revisits locations within that phase-space at each moment in time. Recurrence quantification analysis is used to describe the complexity of a system over time by analyzing small-scale structures in the recurrence plot.

There are a few key concepts underlying the generation of recurrence plots from time series data. In a recurrence plot, the time series data will sit on a plot with axes of time by time. A point $(i, j)$ is plotted if the value at time $i$ and time $j$ are sufficiently 
similar — that is, recurrent — within a specified neighborhood size of the N-dimensional state-space ${ }^{26}$. The state-space of a dynamical system is the vector of possible combinations of states in some number of observable and unobservable dimensions. In order to determine which points are recurrent, it is necessary to reconstruct the higher dimensional phase space of the system. State-space reconstruction is done by embedding the original time series against a time lagged copy of itself ${ }^{27}$. Each time lagged copy is an additional embedding dimension within the state-space ${ }^{28}$. Takens' theorem (1981) shows that the coupling of activity between dimensions preserves the information dynamics of the system as a whole in any single dimension. Put another way, because the subcomponents of a complex dynamical system are intrinsically interdependent, a measurement taken from any one observable subcomponent encodes information from every other (potentially unobservable) subcomponent in the system. Thus, reconstruction of the N-dimensional state-space from a single measured time series allows us to infer the topological dynamics of a multivariate system because the influence of higher dimensional dynamics is encoded in the measured dimension ${ }^{29}$

The logic of state-space reconstruction and embedding within higher dimensions is important for evaluating the dynamics of a natural complex system. The dynamics of natural systems such as crowd behavior, sounds within a piece of music, or even weather patterns, contain $\mathrm{N}$ possible state variables as well as $\mathrm{N}$ possible combinations of nonlinear and bidirectional interactions. State-space reconstruction allows us to infer these unmeasured or unobservable higher order dynamical variables from a single measured variable, in order to evaluate the characteristic dynamics of a system's behavior over time. For a weather system, we might measure the flow of high- and low-pressure systems to evaluate the transitions from stable, 'good' weather to instability that precedes a storm. In a crowd or a musical performance, we might measure movement or acoustic signal to investigate the higher order dynamics of transitions between periods of instability and incoherence to periods of stability and coordination. Here, we take a simple global measure of acoustic signal recorded from a musical performance. This performance demonstrates an orchestrated (non-emergent) transition from uncoordinated to coordinated interaction (described in more detail below). We use RQA to investigate the patterns of coordination in the audio signal as represented in small-scale structures within recurrence plots. We focus on five key measures: recurrence rate, determinism, entropy, average diagonal length, and laminarity.

\section{Music and multi-agent human intearction: A model from acoustic data}

\section{Musical ensembles as models of human social interaction}

Music provides an ideal model system of human social interaction by providing a balance between ecological validity of the social interaction and experimental control(p111) ${ }^{4}$. Analysis of social interactions in musical performance are aided by a "script-like description of the interaction" via the musical score that can be manipulated or referenced by researchers examining the behavioral dynamics of the interaction (p112) ${ }^{4}$. Applying methods of Granger-causality to motion capture data of individual musicians within a performing string quartet can be used to investigate how predictive the history of behavior of one musician is for the future behavior of another. The bodily sway dynamics of these interacting musicians carries Granger-causal information about leader and follower behavior of each musician ${ }^{6}$, and Granger-coupling of bodily sway also carries information about the joint emotional expression and perceived emotional intensity of a musical ${ }^{8}$. Even without reference to a strict musical score, studies of musical interaction have provided insight into how we anticipate and adapt to the behavior of other individuals. Nonlinear analysis techniques have revealed spontaneous self-organizing patterns of coordination across a variety of timescales during joint musical improvisation without a strict score. In a series of experiments analyzing interactions between improvising musicians, Walton et al. describe how behavior produced and received from both the kinesthetic and sonic domain serves to influence and constrain mutual improvisers from the lens of complex dynamical systems $^{5,7}$. Using cross-wavelet spectral analysis and Recurrence Quantification Analysis, Walton et al. describe how mutual behavioral constraints enable an improvising ensemble to produce more complex patterns than any individual would otherwise $^{5,7}$. This mutual interaction establishes a single synergetic system at the level of the improvising group, rather than a set of individuals behaving as single agents.

Rather than analyzing the individual-level behavior of interacting musicians, we apply dynamical systems analysis specifically RQA - on group-level acoustic data to analyze global patterns of coordination in a musical performance. We chose to analyze this performance because it enacts a phenomenological simulation (described below) of the transition from the uncoordinated behavior of individuals to coordinated group behavior that mimics naturalistic multi-agent human interaction.

The composition "Welcome to the Imagination World" composed by Daisuke Shimizu for wind orchestra serves as the model system for multi-agent human interaction. Specifically, the interaction of interest is the shift in dynamics from an uncoordinated, incidental collection of musicians, to a coordinated, interacting ensemble. This transition from uncoordinated, to coordinated interaction is evident in the phenomenological experience of attending (or indeed, performing) this piece of music. The audience will note that, at first, the musicians on stage have no conductor. They sound and look like they are each playing their individual warm up routine. This is because, in fact, the musicians' score tells them to play at random. The composer wanted the sound to be aleatoric, or to occur by chance without being strictly composed. This uncoordinated soundscape 
continues until a melodic pattern starts to emerge from a few of the musicians, still in the absence of a conductor, and still not appearing to be coordinating with the other performers. Next, the "conductor walks on stage [as] the horn, tenor and bass instruments unify into a majestic introduction", according to program notes from the composer (windrep.org). This marks the transition from the uncoordinated actions of individual musicians to the coordinated ensemble musicianship the audience expects. The remaining musical score is composed to dictate the acoustic interaction of the musicians on stage. Thus, the rest of the performance demonstrates the coordinated interactions of an interdependent complex system: the multi-agent musical ensemble.

\section{Results}

\section{Recurrence Quantification Analysis}

Figures 1 and 2 show recurrence plots generated from the time series data of the recording. The recurrence plots visualize the characteristic patterns of recurrence which are then quantified through recurrence quantification analysis. Figure 1 displays recurrence plots for the first and last 30 seconds of each Coordination Category (Uncoordinated or Coordinated). Darker segments of the recurrence plots indicate the presence of more recurrent data points. Vertical and horizontal lines indicate periods of stability in the system, where one state was visited for a period of up to a few seconds at a time.

The recurrence plots in Figure 2 are representative 5-second samples drawn from each 30 second sample in Figure 1. These shorter samples are labeled as the associated Performance Event the 5-second sample is drawn from. Note that the 'Introduction' section falls in the middle of the performance, marking the shift from the Uncoordinated to Coordinated interaction among the musicians, and thus the order of Performance Events is: 1. Aleatoric, 2. Transition, 3. Introduction, 4. Finale. Increased presence of diagonal lines and vertical structures in the Introduction and Finale sections indicate increasingly coordinated interaction among the musicians. The diagonal striping in the Introduction indicates some periodicity in the signal, similar to the periodicity of a sine wave, and results in this case from a(n almost) unison chord during those few seconds. The Aleatoric and Transition plots at the top more closely resemble white noise, with fewer recurrent points, shorter diagonals, and less apparent vertical structures.

Recurrence Quantification Analysis quantifies the qualitative patterns observed in these recurrence plots. To describe the behavior of our model multi-agent system, five RQA measures were evaluated. The first RQA measures evaluated are common measures of recurrence: a) patterns that repeat over time (\%recurrence, the percentage of recurrent points on the recurrence plot); b) behaviors that belong to a longer sequence of behavior (\%determinism, or the percentage of points that fall on any diagonal line in the recurrence plot); c) the amount of disorder there is in these sequences (entropy, or the variability in lengths of these diagonal lines). Additional patterns of stability in the system's behavior were measured by examining d) clusters of behavior (\%laminarity, or the percentage of points that fall on a vertical line in the recurrence plot), and e) the average length of time our multi-agent system stays in one behavioral pattern (average diagonal length, the average length of diagonal lines). Average diagonal line length is a measure related to determinism. Longer average diagonal lines reflect the stability of a system by indicating longer, more continuous states. Similarly, higher laminarity shows the rigidity, or 'stickiness' of a system that stays in one or more states of a behavior for a length of time (Davis et al.).

Higher values and increased variability were evident for most RQA measures for Performance Events within the Coordinated sections of the performance vs Uncoordinated sections (Figure 3). However, distribution plots do not readily visualize different trajectories of behavior over time. Varying trajectories of each RQA measure for Coordinated vs Uncoordinated sections of the performance is evident in the serial plots in Figure 4. The majority of RQA values hover around a single value over time during the Uncoordinated Performance Events (Aleatoric and Transition), indicating little interaction among musicians-the agents in our model system. As interactive behavior emerges among musicians, the joint activity of the interacting ensemble in the Coordinated Performance Events (Introduction and Finale) begin to show increased recurrent points overall (Recurrence Rate), with emerging presence of longer sequences of behavior as represented by higher levels of Determinism, and higher levels of Entropy indicating more variability in sequence length. Increased values of Laminarity indicate enhanced stability in the system. The intermittency of these stable periods in the Coordinated Performance Events as shown in the recurrence plots can also be seen in the varying high and low values of Laminarity over time. 

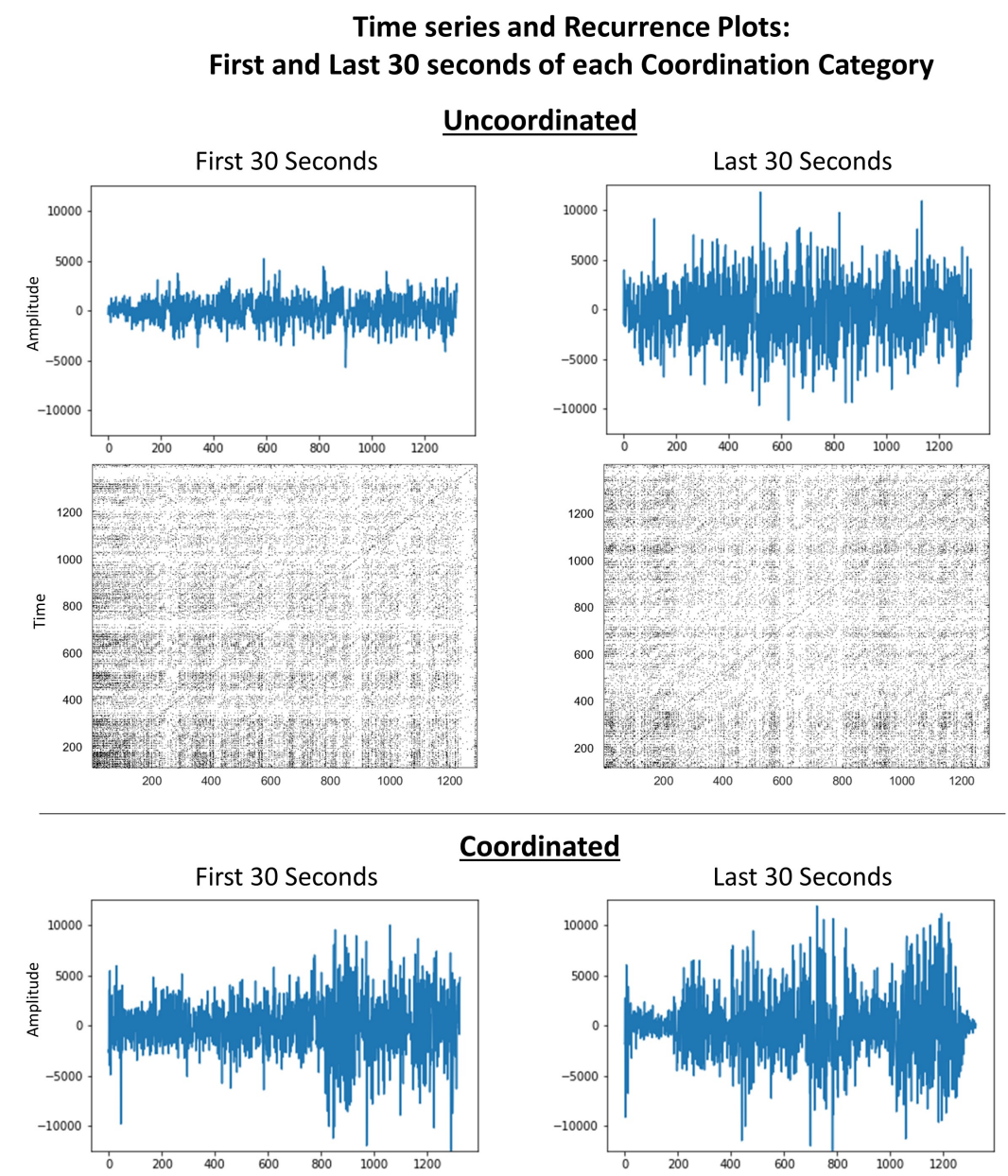

Coordinated
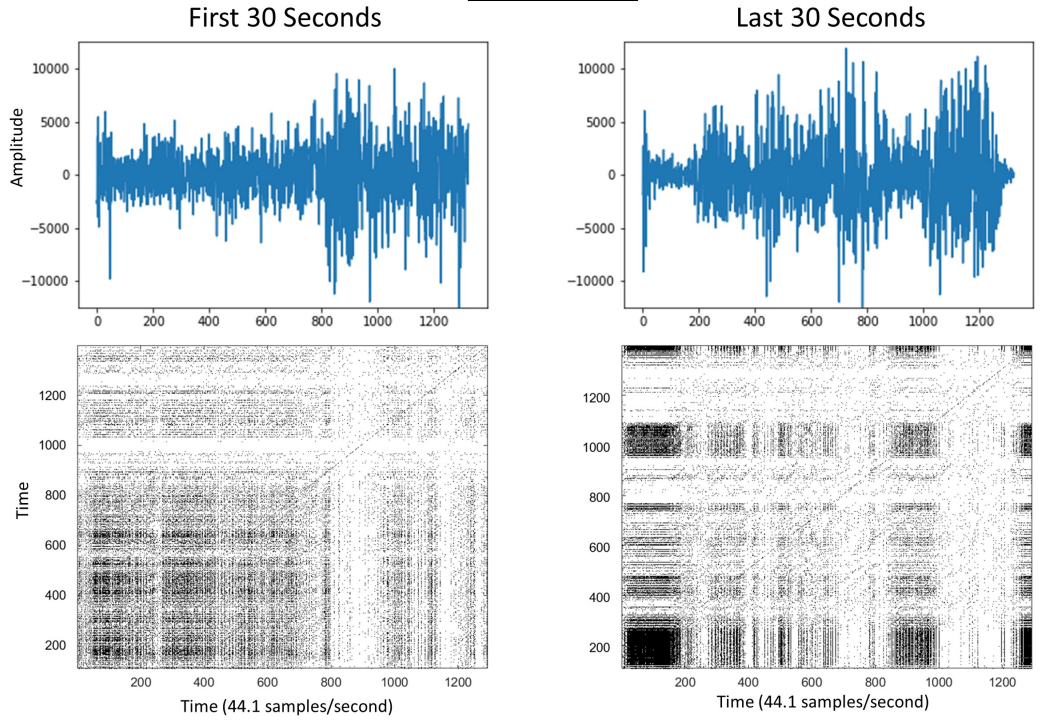

Figure 1. Time Series and Recurrence Plots for the first and last 30 seconds of each Coordination Category (Uncoordinated or Coordinated). Darker segments of the recurrence plots indicate the presence of more recurrent data points. Vertical and horizontal lines indicate periods of stability in the system, where one state was visited for a period of up to a few seconds at a time.

\section{Statistical Analysis}

Statistical analysis was performed on the first and last 25 samples of each Coordination Category, representing the first and last 30 seconds each of Uncoordinated and Coordinated sections of the piece. Percent recurrence (\%REC) for the first thirty seconds of each category was highest in coordinated (mean 0.093/ sd 0.030) when compared to uncoordinated (mean 0.081/ sd 0.009). \%REC for Performance Event showed a slight decrease from Aleatoric (mean $0.085 / \mathrm{sd} 0.009$ ) to Transition (mean 0.078 / sd 0.008) and increased in both Introduction (mean 0.089/ sd 0.017) and Finale (mean 0.098 /sd 0.039) (Figure 3 A). 
Time series and Recurrence Plots:

5 seconds of each Performance Event

Uncoordinated
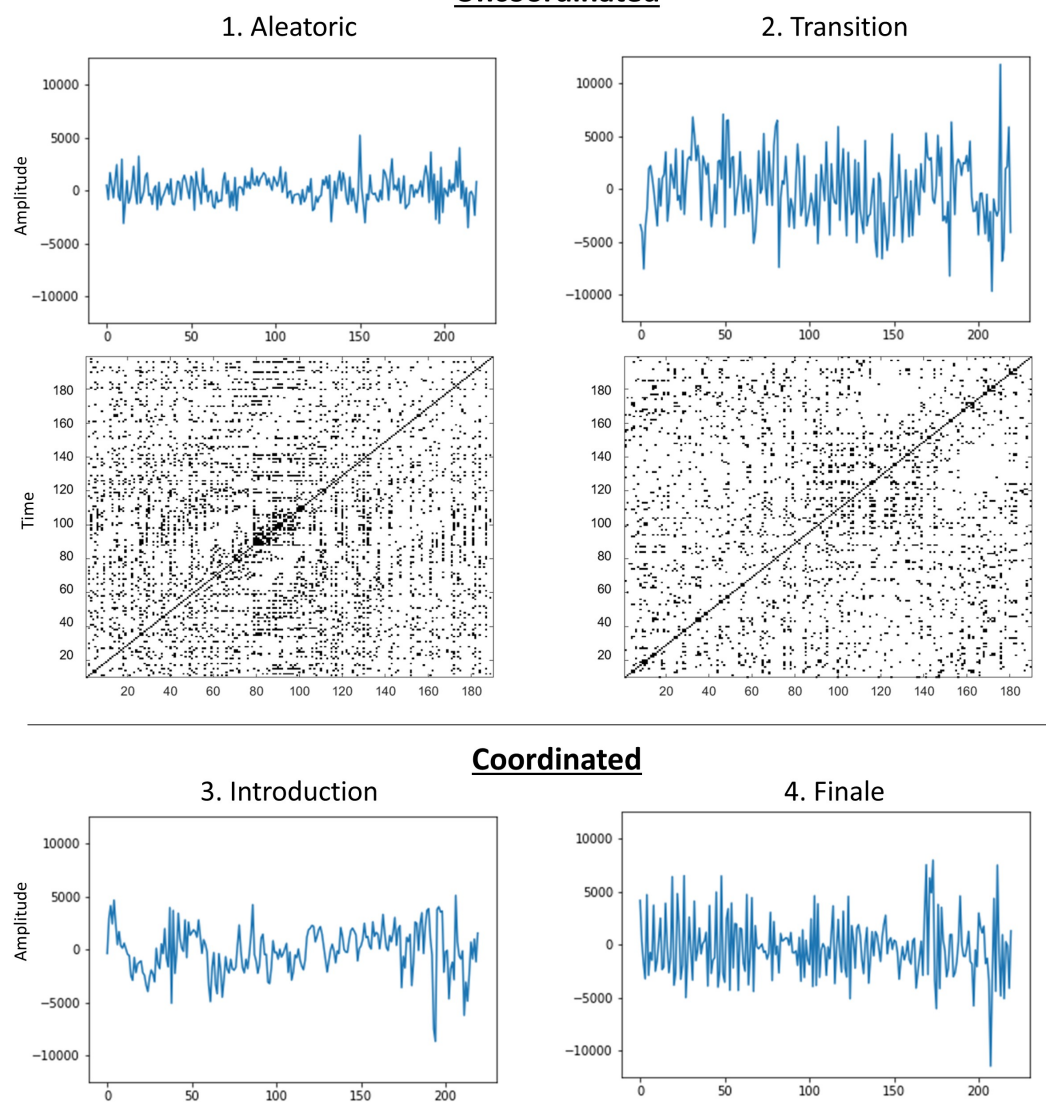

\section{Coordinated}
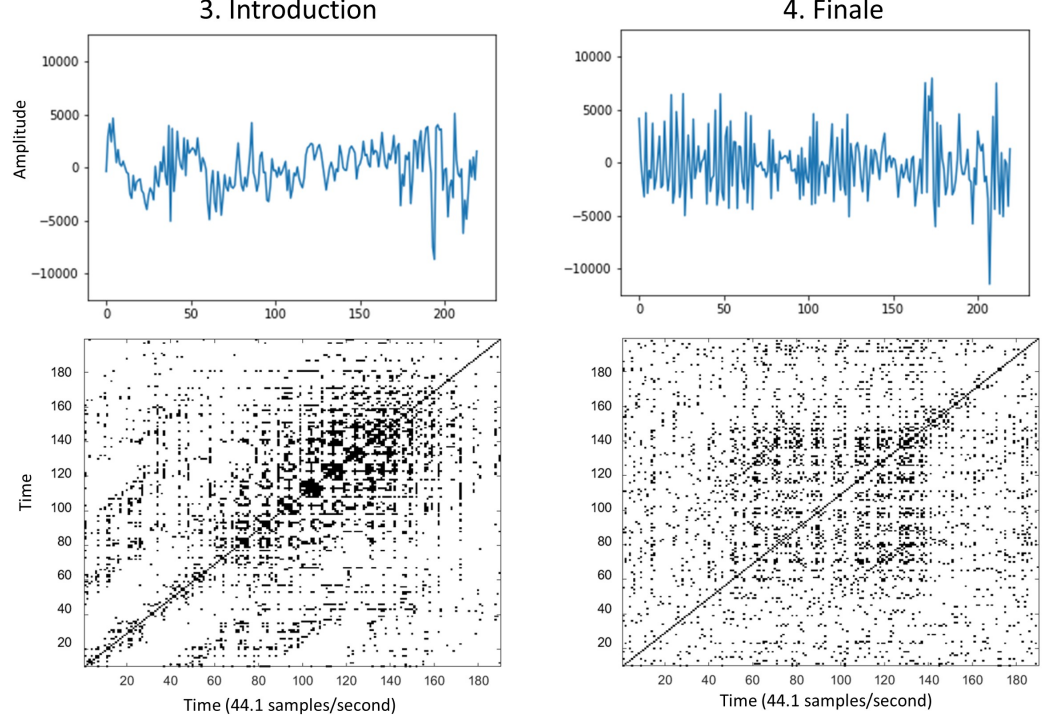

Figure 2. Time Series and Recurrence Plots for representative 5-second samples drway from each 30-second sample in Figure 1, and labeled as the associated Performance Event the 5-second sample is drawn from. Uncooridnated Aleatoric and Transition plots more closely resemble white noise, with fewer recurrent points, shorter diagonals, and less apparent vertical structures. Increased presence of diagonal lines and vertical structures in Coordinated Introduction and Finale plots indicate increasingly coordinated interaction among the musicians.

Percent determinism (\%DET) for the first thirty seconds of each category was, highest in the coordinated condition (mean $0.277 /$ sd 0.147) when compared to uncoordinated (mean 0.163/ sd 0.024).\%DET for Performance event shows the same pattern, with a slight decrease from Aleatoric (mean 0.167 /sd 0.023) to Transition (mean 0.160 / sd 0.025), and increases in both Introduction (mean 0.266/ sd 0.106) and Finale (mean 0.288 /sd 0.179) (Figure 3, B). Entropy for the first thirty seconds of each category was highest in the coordinated condition (mean 0.693/ sd 0.432) when compared to uncoordinated (mean $0.295 /$ 


\section{Distribution of RQA Measures for Uncoordinated and Coordinated Performance Events}
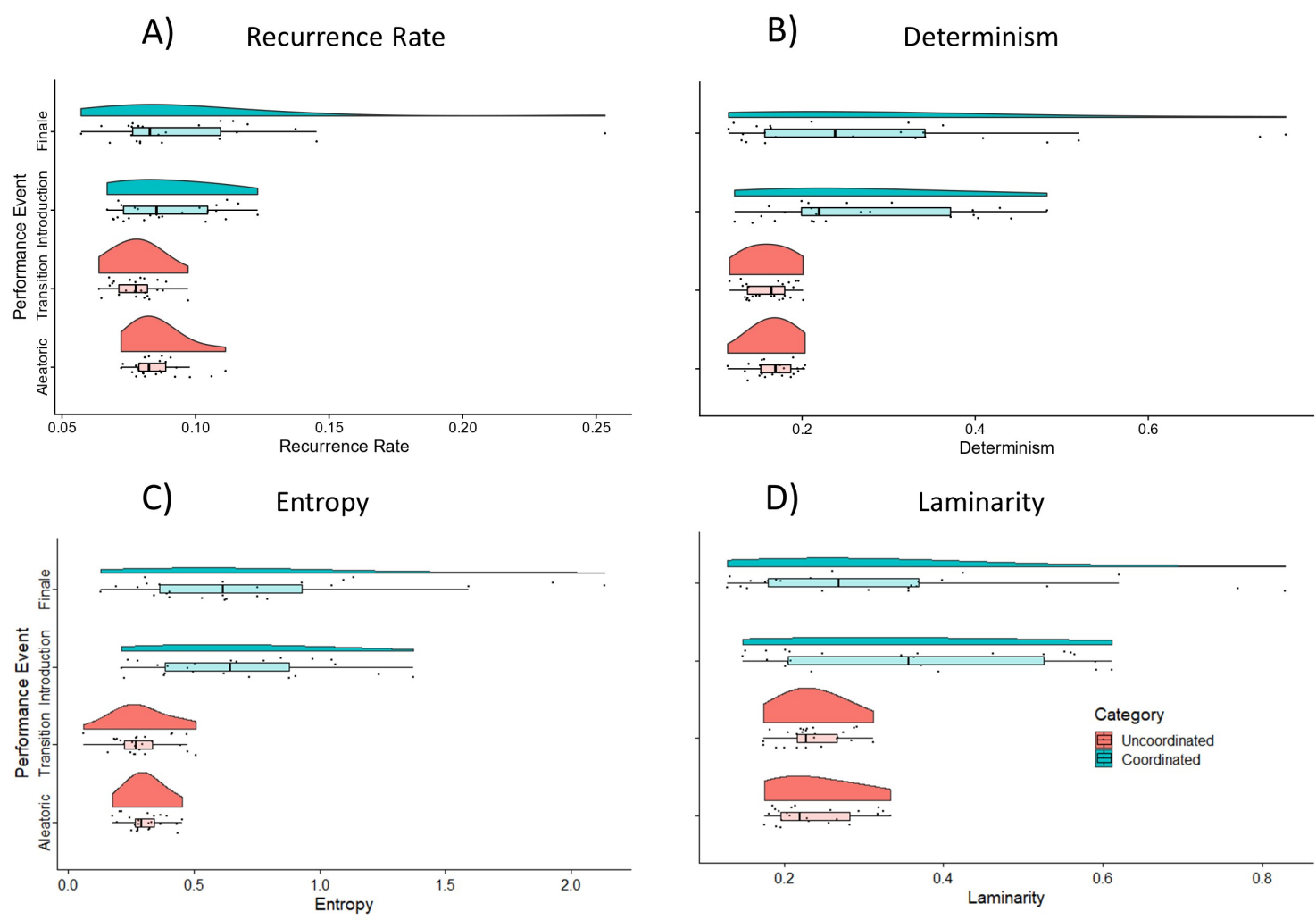

Figure 3. Raincloud Plots show higher levels, and wider variance, in each RQA metric in Coordinated compared to Uncoordinated Categories. Boxplots show sample median and interquartile range.

sd 0.091). Entropy for Performance event shows the same pattern, with a slight decrease from Aleatoric (mean $0.305 / \mathrm{sd} 0.074$ ) to Transition (mean 0.292 / sd 0.110), and increases in both Introduction (mean 0.654/ sd 0.325) and Finale (mean $0.731 / \mathrm{sd}$ 0.521) (Figure 3, C.) Laminarity for the first thirty seconds of each category was highest in the coordinated condition (mean $0.340 / \mathrm{sd} 0.175$ ) when compared to uncoordinated (mean 0.236/ sd 0.045).Laminarity for Performance event shows the same pattern, with a slight decrease from Aleatoric (mean 0.240/ sd 0.053) to Transition (mean 0.234 / sd 0.037), and increases in both Introduction (mean 0.353/ sd 0.162) and Finale (mean 0.326/sd 0.190) (Figure 3, D).

Higher values and increased variability were evident for most RQA measures for Performance Events within the Coordinated sections of the performance vs Uncoordinated sections (Figure 3). However, distribution plots do not readily visualize different trajectories of behavior over time. Varying trajectories of each RQA measure for Coordinated vs Uncoordinated sections of the performance is evident in the serial plots in (Figure 4). The majority of RQA values hover around a single value over time during the Uncoordinated Performance Events (Aleatoric and Transition), indicating little interaction among musicians-the agents in our model system. As interactive behavior emerges among musicians, the joint activity of the interacting ensemble in the Coordinated Performance Events (Introduction and Finale) begin to show increased recurrent points overall (Recurrence Rate), with emerging presence of longer sequences of behavior as represented by higher levels of Determinism, and higher levels of Entropy indicating more variability in sequence length. Increased values of Laminarity indicate enhanced stability in the system. The intermittency of these stable periods in the Coordinated Performance Events as shown in the recurrence plots can also be seen in the varying high and low values of Laminarity over time.

To further examine the trends above, Linear Mixed Effects models (LMEs) were applied to determine the differential effect of Coordination Category and Performance Event on each RQA measure of interest. Log \%REC was predicted by Coordination 


\section{RQA Measures for each Performance Event over 25 consecutive five-second samples}

A)

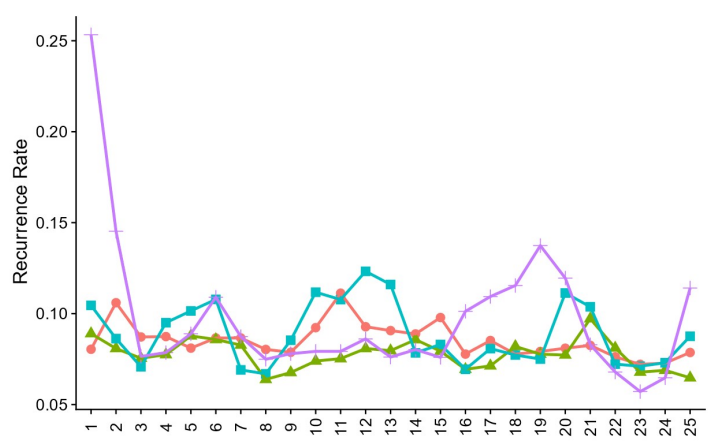

C) Entropy

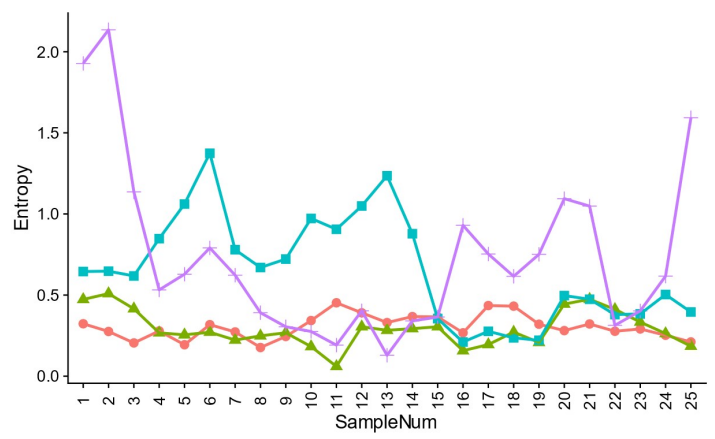

B)

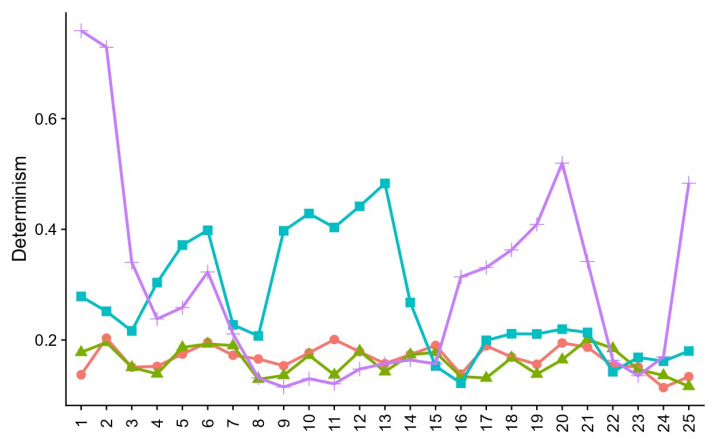

D)

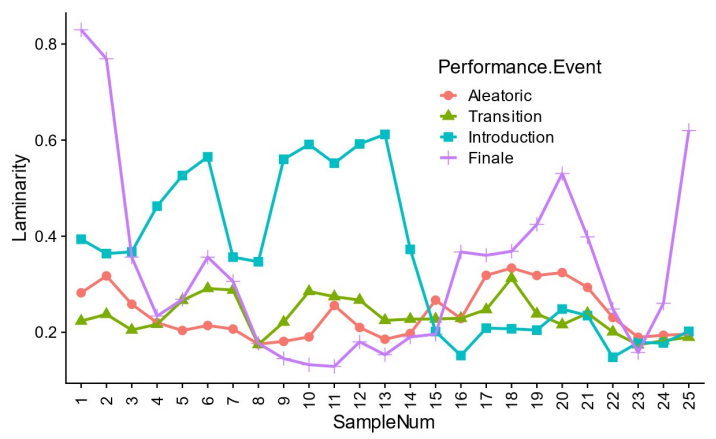

Figure 4. Serial plots visualizing the trajectories of recurrence behaviors over time. A) Coordinated Performance Events (Introduction and Finale) show increased recurrent points overall (Recurrence Rate) compared with Uncoordinated Performance Events (Aleatoric and Transition). B) Emerging presence of longer sequences of behavior as represented by higher levels of Determinism, and C) higher levels of Entropy in Coordinated Performance Events, indicating more variability in sequence length. D) Increased values of Laminarity in Coordinated Performance Events indicate enhanced stability in the system. The intermittency of these stable periods in the Coordinated Performance Events as shown in the recurrence plots (Figures 1 and 2) can also be seen in the varying high and low values of Laminarity over time in the serial plots.

Category $\left(\chi^{2}(1)=8.0805, \mathrm{p}=0.0045\right)$, and by Performance Event $\left(\chi^{2}(3)=12.594, \mathrm{p}=0.0056\right)$. Log \%DET was predicted by Coordination Category $\left(\chi^{2}(1)=30.455, \mathrm{p}=3.418 \mathrm{e}-8\right)$, and by Performance Event $\left(\chi^{2}(3)=30.656, \mathrm{p}=1.004 \mathrm{e}-6\right)$. Log Entropy was predicted by Coordination Category $\left(\chi^{2}(1)=41.261, p=1.332 \mathrm{e}-10\right)$, and by Performance Event $\left(\chi^{2}(3)=41.739\right.$, $\mathrm{p}=4.557 \mathrm{e}-9) . \log$ Laminarity was predicted by Coordination Category $\left(\chi^{2}(1)=10.382, \mathrm{p}=.0013\right)$, and by Performance Event $\left(\chi^{2}(3)=11.516, p=0.0092\right)$.

LME model comparison results are reported in Table 1 for log transformations of each RQA measure, except for Average Diagonal Length because assumptions of normality/heterocedasticity were not met. LMEs with a fixed effect of Coordination Category showed lower AIC and BIC values than the null model, or the model with a fixed effect of Performance Event, indicating that RQA measures are better predicted by the Coordination Category (Uncoordinated vs Coordinated) of each sample than by the Performance Event (Aleatoric, Transition, Introduction, and Finale-which are smaller subdivisions of each Coordination Category). Table 1. Linear mixed effects model results. Models were fixed effect of interest (Coordination Category or Performance Event) with random effect of Sample Number, against a random intercept model without the fixed effect in question. Models reveal differential effect of the fixed effect of interest on the log transformed RQA measure of interest (Recurrence Rate, Determinism, Entropy, or Laminarity). 
Table 1. Linear Mixed Effects models evaluating the effect of Coordination Category and Performance Event on each RQA Measure of Interest

\begin{tabular}{|c|c|c|c|c|c|c|}
\hline \multirow{3}{*}{ Fixed Effects } & \multicolumn{3}{|c|}{ Recurrence Rate } & \multicolumn{3}{|c|}{ Determinism } \\
\hline & Full Model & & Null Model & Full Model & & Null Model \\
\hline & Estimate & SE & & Estimate & SE & \\
\hline \multicolumn{7}{|l|}{ Coordination Category } \\
\hline Uncoordinated (Intercept) & -2.514 & 0.0304 & & -1.8225 & 0.0503 & \\
\hline Coordinated & 0.1052 & 0.0360 & & 0.4217 & 0.0703 & \\
\hline \multicolumn{7}{|l|}{ Goodness of Fit } \\
\hline Deviance & -43.82 & & -35.74 & 75.801 & & 106.256 \\
\hline AIC & -35.82 & & -29.74 & 83.801 & & 112.256 \\
\hline BIC & -25.4 & & -21.924 & 94.222 & & 120.071 \\
\hline$\chi^{2}(\mathrm{df})$ & $8.0805(1) * *$ & & & $30.455(1)^{* * *}$ & & \\
\hline \multicolumn{7}{|l|}{ Performance Event } \\
\hline Aleatoric (Intercept) & -2.4678 & 0.0389 & & -1.8006 & 0.0706 & \\
\hline Transition & -0.0924 & 0.049 & & -0.0439 & 0.0993 & \\
\hline Introduction & 0.0324 & 0.0494 & & 0.4039 & 0.0993 & \\
\hline Finale & 0.0856 & 0.0494 & & 0.3956 & 0.0993 & \\
\hline \multicolumn{7}{|l|}{ Goodness of Fit } \\
\hline Deviance & -48.334 & & -35.74 & 75.599 & & 106.256 \\
\hline AIC & -36.334 & & -29.74 & 87.599 & & 112.256 \\
\hline BIC & -20.703 & & -21.924 & 103.23 & & 120.07 \\
\hline \multirow[t]{2}{*}{$\chi^{2}(\mathrm{df})$} & $12.594(3) * *$ & & & $30.656(3)^{* * *}$ & & \\
\hline & & Entropy & & & minarity & \\
\hline \multirow[t]{2}{*}{ Fixed Effects } & Full Model & & Null Model & Full Model & & Null Model \\
\hline & Estimate & SE & & Estimate & SE & \\
\hline \multicolumn{7}{|l|}{ Coordination Category } \\
\hline Uncoordinated (Intercept) & -1.2647 & 0.0709 & & -1.4567 & 0.0535 & \\
\hline Coordinated & 0.7169 & 0.1003 & & 0.2506 & 0.0758 & \\
\hline \multicolumn{7}{|l|}{ Goodness of Fit } \\
\hline Deviance & 145.78 & & 187.04 & 89.655 & & 100.037 \\
\hline $\mathrm{AIC}$ & 153.78 & & 193.04 & 97.655 & & 106.037 \\
\hline BIC & 164.2 & & 200.86 & 108.08 & & 113.85 \\
\hline$\chi^{2}(\mathrm{df})$ & $41.261(1)^{* * *}$ & & & $10.382(1)^{* *}$ & & \\
\hline \multicolumn{7}{|l|}{ Performance Event } \\
\hline Aleatoric (Intercept) & -1.2167 & 0.1001 & & -1.4504 & 0.0753 & \\
\hline Transition & -0.0961 & 0.1415 & & -0.0127 & 0.1065 & \\
\hline Introduction & 0.6593 & 0.1415 & & 0.3008 & 0.1065 & \\
\hline Finale & 0.6783 & 0.1415 & & 0.1877 & 0.1065 & \\
\hline \multicolumn{7}{|l|}{ Goodness of Fit } \\
\hline Deviance & 145.3 & & 187.04 & 88.521 & & 100.037 \\
\hline AIC & 157.3 & & 193.04 & 100.52 & & 106.04 \\
\hline BIC & 172.94 & & 200.86 & 116.15 & & 113.85 \\
\hline$\chi^{2}(\mathrm{df})$ & $41.739(3)^{* * *}$ & & & $11.516(3) * *$ & & \\
\hline
\end{tabular}

Note. ${ }^{* * *} \mathrm{p}<0.001, * * \mathrm{p}<0.01,{ }^{*} \mathrm{p}<0.05$

\section{Discussion}

The current study evaluated a musical performance which enacted a real-life simulation of the transition from uncoordinated to orchestrated (non-emergent) coordinated behavior. That is, the musicians in this ensemble simulated the transition from 
disorder to order in one form of social interaction-a musical performance-by following the orchestration of the musical score, accentuated by the presence of a conductor on stage at just the time the musicians begin to play music together as a single interacting ensemble.

We have empirically demonstrated differences in the coordination patterns of this originally non-interacting collection of independent musicians versus their collective dynamics as an interdependent group of musicians participating in a joint musical interaction. Thus, this study is one example of how conceptualizing and evaluating musical interaction using the tools of coordination dynamics and dynamical systems theory can reveal insights into the self-organizing behavior which underlies multi-agent musical interaction (see Schiavio et al, 2021 for a thorough review ${ }^{30}$ ).

Unlike previous studies of human social interaction, this study evaluated acoustic data of a musical performance to infer the global behavior of musicians in the performing ensemble. This means we did not have access to the behavior of individual musicians in order to evaluate correlations between individuals. Instead, we relied on recurrence features extracted from global acoustic data and represented in recurrence plots. The uncoordinated behavior of individual, non-interacting musicians at the start of the performance demonstrated lower measures of recurrence and stability in the group-level acoustic data. When the musicians began interacting with each other as a coordinated musical ensemble, recurrence and stability measures increased overall. The interaction served as a coupling mechanism for this multi-agent human group, just as the physical platform served as a coupling mechanism for the metronome group. In addition, the interacting ensemble also exhibited further variability in these recurrence measures. This indicates that the interacting ensemble of musicians were able to explore a greater variety of behavior than when they performed as a stage full of individual musicians.

When the function of each musician becomes interdependent with the functions of the other musicians, the group becomes a complex system. The collective cognition (c.f. ${ }^{31,32}$ ) that takes place in order to generate the coordinated music makes the group function a little bit like one large mind ${ }^{33,34}$ (c.f. ${ }^{35}$ ). Thus, when that collective mind has an audio time series extracted from it (that is subjected to state-space reconstruction), it can provide insight into how and when those individual subcomponents achieve their coordinated behavior.

In addition, this musical performance is a model system for other forms of multi-agent human interaction. The local rules that govern the emergence of interaction in this ensemble arise from a musical score, which stipulates when the musicians must begin performing as an interacting group, as well as a conductor who acts as a leader during the coordinated section of musical performance. However, RQA applied to this model system provides an upper bound for what recurrence dynamics to expect in truly emergent coordination in multi-agent human interaction in the wild-perhaps in less orchestrated (i.e. more improvised) forms of musical interaction such as leaderless interaction in free jazz improvisation ${ }^{36}$, and even day-to-day social dynamics extending beyond musical interaction, such as walking in groups ${ }^{37}$ or interacting in large crowds.

Extending the current analysis methods to other forms of multi-agent human interaction will also expand current knowledge regarding the affective dynamics of acoustic and motor coordination during social interaction. Listening to music while moving in time with a partner increases perceived connectedness among a dyad ${ }^{38}$. Movement synchrony in dancers increases affiliation with the group ${ }^{39}$ and can increase affective engagement from an audience ${ }^{40}$. In a dot-motion paradigm, velocity-based synchrony (associated with expert interaction) in comparison with interval-based synchrony (associated with novice interaction) from ostensibly improvising performers is rated by observers as more beautiful, and the 'performers' are judged to like each other more ${ }^{41}$. The affiliatory effects of synchronous interaction are not always positive, however, and can actually lead to increased compliance with requests to engage in aggressive behavior ${ }^{42}$. Multi-agent groups in the wild, such as crowds at a sporting event or gathering for a protest, may not be engaging in strictly synchronous motor coordination, however their acoustic behavior may exhibit measurable patterns of distributed coordination which influence the affective states of the group and individual. A question remains as to what are the recurrence dynamics of acoustic behavior of multi-agent groups in the wild, and what role does coordinated acoustic behavior play in the affective dynamics of individuals engaging in or observing these social interactions.

\section{Methods}

\section{Extracting Acoustic Data}

An audio recording of 'Welcome to the Imagination World' was obtained from the 2009 performance by the Inagauken Wind Orchestra posted on YouTube. An MP3 was downloaded using the YouTube to Mp3 video converter. The audio recording was labeled by two independent raters with terminal music degrees and substantial training in music theory. Raters were familiarized with the program notes for the composition (retrieved from www.windrep.org) and were instructed to identify where the musicians' transition from "random ad lib" to "unify[ing] majestic introduction" as described in the program note from the composer (operationalized as uncoordinated and coordinated, respectively), as well as noting any details of the performance they found important. The audio was subsequently labeled into two Coordination Categories: Uncoordinated and Coordinated. The first and last 30-seconds of each Coordination Category (Uncoordinated or Coordinated) were also labeled 
into four Performance Event subcategories (Aleatoric, Transition, Introduction, and Finale), two in each Coordination Category, respectively.

The audio recording was converted from stereo to mono in Audacity 2.3.0, converted from an MP3 to a WAV file, removed DC offset, and normalized to -1.0 dBFS. Python 3.7 in Jupyter Notebooks was used to downsample the audio from $44.1 \mathrm{KHz}$ to $44.1 \mathrm{~Hz}$, and to create separate time series for each labeled Coordination Category. Time series containing only audience noise, or dominated by audience noise, were discarded. The time series for each performance event category was then extracted into 5 -second overlapping windows, sliding by 1 second at a time, saving only full 5 -second samples.

\section{Recurrence Quantification Analsys}

The CRP toolbox in MATLAB 2018b was used to visualize the acoustic time series as recurrence plots and to carry out RQA (Marwan; Marwan et al.). RQA parameters were set with an embedding dimension of 4, delay of 10, neighborhood size (radius) of 1standard deviation, using maximum norm to calculate neighbors of the phase space trajectory.

\section{Statistical Analysis}

Statistical analysis was performed on the first and last 25 samples of each Coordination Category, representing the first and last 30 seconds each of Uncoordinated and Coordinated sections of the piece. Raincloud plots ${ }^{43}$ and serial time series plots to visualize distributions and trajectories of the RQA measures were created in RStudio 1.1.463 using ggplot $2^{44}$.

Linear Mixed Effects models (LMEs) were applied to determine the differential effect of Coordination Category and Performance Event on each RQA measure of interest. LMEs were calculated using the lme 4 package ${ }^{45}$. The first model examined the effects of Coordination Category on each RQA measure, with a fixed effect of Coordination Category and random effects of Sample Number, to account for any variance arising from individual five-second samples. The second model examined the effects of Performance Event on each RQA measure, with a fixed effect of Performance Event and random effects of Sample Number.

- Full model with fixed effect of Category (Coordinated vs Uncoordinated) and random effects of order (sample number):

$$
\begin{aligned}
\text { RQAMetric }_{i} & \sim N\left(\alpha_{j[i]}+\beta_{1} \text { (Category), } \sigma^{2}\right) \\
\alpha_{j} & \sim N\left(\mu_{\alpha_{j}}, \sigma_{\alpha_{j}}^{2}\right), \text { for SampleNum } \mathrm{j}=1, \ldots, \mathrm{J}
\end{aligned}
$$

- Null, intercept-only model without the fixed effect of Category:

$$
\begin{aligned}
\text { RQAMetric }_{i} & \sim N\left(\alpha_{j[i]}, \sigma^{2}\right) \\
\alpha_{j} & \sim N\left(\mu_{\alpha_{j}}, \sigma_{\alpha_{j}}^{2}\right), \text { for SampleNum } \mathrm{j}=1, \ldots, \mathrm{J}
\end{aligned}
$$

- Full model with fixed effect of Performance Event (Aleatoric, Transition, Introduction, Finale) and random effects of order:

$$
\begin{aligned}
\text { RQAMetric }_{i} & \sim N\left(\alpha_{j[i]}+\beta_{1}(\text { Performance. Event }), \sigma^{2}\right) \\
\alpha_{j} & \sim N\left(\mu_{\alpha_{j}}, \sigma_{\alpha_{j}}^{2}\right), \text { for SampleNum } \mathrm{j}=1, \ldots, \mathrm{J}
\end{aligned}
$$

- Null, intercept-only model without the fixed effect of Performance Event:

$$
\begin{aligned}
\text { RQAMetric }_{i} & \sim N\left(\alpha_{j[i]}, \sigma^{2}\right) \\
\alpha_{j} & \sim N\left(\mu_{\alpha_{j}}, \sigma_{\alpha_{j}}^{2}\right), \text { for SampleNum } \mathrm{j}=1, \ldots, \mathrm{J}
\end{aligned}
$$

LMEs with random intercepts such as this are robust to variability in individual subjects, or five-second samples in this case. This is because random intercept models assume a different baseline-level of the RQA measure of interest in each fixed effect for each sample, thus accounting for any differences that may appear by virtue of the sequential order of obtaining each sample. Mixed models also address issues of non-independence due to inherent correlations between successive samples of musical performance dat ${ }^{46}$. Goodness of fit was evaluated by model comparison of the full models against null, intercept only models without the fixed effect in question, as shown in the equations above. Four of the five RQA measures of interest were modeled (\%recurrence, \%determinism, entropy, and laminarity). LME results based on Average Diagonal Length are not reported. Residuals plots revealed that the LMEs for Average Diagonal Length did not meet criteria for assumptions of normality and heteroscedasticity, even after log transformation, and as such were not a good model for the data. Statistical significance was obtained by computing a likelihood ratio test of the full model to a null model without the fixed effect in question. 


\section{References}

1. Meyer, L. Emotion and Meaning in Music (University of Chicago Press, 1956).

2. Huron, D. Sweet Anticipation: Music and the Psychology of Expectation (MIT Press, 2008).

3. Juslin, P. N. \& Västfjäll, D. Emotional responses to music: the need to consider underlying mechanisms. Behav. Brain Sci. 31, 559-621, DOI: 10.1017/S0140525X08005293 (2008).

4. D’Ausilio, A., Novembre, G., Fadiga, L. \& Keller, P. E. What can music tell us about social interaction? Trends Cogn. Sci. 19, 111-114, DOI: 10.1016/j.tics.2015.01.005 (2015).

5. Walton, A. E., Richardson, M. J., Langland-Hassan, P. \& Chemero, A. Improvisation and the self-organization of multiple musical bodies. Front. Psychol. 06, DOI: 10.3389/fpsyg.2015.00313 (2015).

6. Chang, A., Livingstone, S. R., Bosnyak, D. J. \& Trainor, L. J. Body sway reflects leadership in joint music performance. Proc. Natl. Acad. Sci. 114, E4134-E4141, DOI: 10.1073/pnas.1617657114 (2017).

7. Walton, A. E. et al. Creating Time: Social Collaboration in Music Improvisation. Top. Cogn. Sci. 10, 95-119, DOI: 10.1111/tops.12306 (2018).

8. Chang, A., Kragness, H. E., Livingstone, S. R., Bosnyak, D. J. \& Trainor, L. J. Body sway reflects joint emotional expression in music ensemble performance. Sci. Reports 9, 205, DOI: 10.1038/s41598-018-36358-4 (2019).

9. Buhl, J. et al. From Disorder to Order in Marching Locusts. Science 312, 1402-1406, DOI: 10.1126/science.1125142 (2006).

10. Cavagna, A. et al. Scale-Free Correlations in Starling Flocks. Proc. Natl. Acad. Sci. 107, DOI: 10.1073/pnas.1005766107 (2010).

11. Néda, Z., Ravasz, E., Brechet, Y., Vicsek, T. \& Barabási, A.-L. The sound of many hands clapping. Nature 403, 849-850, DOI: $10.1038 / 35002660$ (2000).

12. Schmidt, R. \& O'Brien, B. Evaluating the Dynamics of Unintended Interpersonal Coordination. Ecol. Psychol. 9, 189-206, DOI: 10.1207/s15326969eco0903_2 (1997). Publisher: Routledge.

13. Richardson, M. J., Marsh, K. L., Isenhower, R. W., Goodman, J. R. \& Schmidt, R. Rocking together: Dynamics of intentional and unintentional interpersonal coordination. Hum. Mov. Sci. 26, 867-891, DOI: 10.1016/j.humov.2007.07.002 (2007).

14. Abney, D. H., Paxton, A., Dale, R. \& Kello, C. T. Movement dynamics reflect a functional role for weak coupling and role structure in dyadic problem solving. Cogn. Process. 16, 325-332, DOI: 10.1007/s10339-015-0648-2 (2015).

15. Nalepka, P., Kallen, R. W., Chemero, A., Saltzman, E. \& Richardson, M. J. Herd Those Sheep: Emergent Multiagent Coordination and Behavioral-Mode Switching. Psychol. Sci. 28, 630-650, DOI: 10.1177/0956797617692107 (2017).

16. Paxton, A. \& Dale, R. Interpersonal Movement Synchrony Responds to High- and Low-Level Conversational Constraints. Front. Psychol. 8, 1135, DOI: 10.3389/fpsyg.2017.01135 (2017).

17. Richardson, D. C. \& Dale, R. Looking To Understand: The Coupling Between Speakers' and Listeners' Eye Movements and Its Relationship to Discourse Comprehension. Cogn. Sci. 29, 1045-1060, DOI: 10.1207/s15516709cog0000_29 (2005). Publisher: John Wiley \& Sons, Ltd.

18. Chang, A. et al. Body Sway Predicts Romantic Interest in Speed Dating. Soc. Cogn. Affect. Neurosci. 185-192, DOI: 10.1093/scan/nsaa093 (2020).

19. Takens, F. Detecting strange attractors in turbulence. In Rand, D. \& Young, L. (eds.) Dynamical Systems and Turbulence, vol. 898 of Lecture Notes in Mathematics (Springer, Berlin, Heidelberg, Warwick, 180).

20. Vlachos, I. \& Kugiumtzis, D. Nonuniform state-space reconstruction and coupling detection. Phys. Rev. E 82, 016207, DOI: 10.1103/PhysRevE.82.016207 (2010). Publisher: American Physical Society.

21. Shimizu, D. Welcome to the Imagination World (2016). www.windrep.org/Welcome_to_the_Imagination_World.

22. InagakuenWindOrchestra. Welcome to the Imagination World (2009). Inagakuen Wind Orchestra. Youtube, uploaded by kamoshitamoyashi. 17 Feb. 2011, https://www.youtube.com/watch?v=-wJ9ZsgO3QI.

23. Kiverstein, J., Miller, M. \& Rietveld, E. The feeling of grip: novelty, error dynamics, and the predictive brain. Synthese 196, 2847-2869, DOI: 10.1007/s11229-017-1583-9 (2019).

24. Pantaleone, J. Synchronization of metronomes. Am. J. Phys. 70, 992-1000, DOI: 10.1119/1.1501118 (2002). 
25. Francke, M., Pogromsky, A. \& Nijmeijer, H. Huygens' clocks: 'sympathy' and resonance. Int. J. Control. 93, 274-281, DOI: 10.1080/00207179.2019.1590736 (2020). Publisher: Taylor \& Francis.

26. Balasubramaniam, R., Riley, M. A. \& Turvey, M. Specificity of postural sway to the demands of a precision task. Gait \& Posture 11, 12-24, DOI: 10.1016/S0966-6362(99)00051-X (2000).

27. Riley, M., Balasubramaniam, R. \& Turvey, M. Recurrence quantification analysis of postural fluctuations. Gait \& Posture 9, 65-78, DOI: 10.1016/S0966-6362(98)00044-7 (1999).

28. Stephen, D. G., Dixon, J. A. \& Isenhower, R. W. Dynamics of representational change: Entropy, action, and cognition. $J$. Exp. Psychol. Hum. Percept. Perform. 35, 1811-1832, DOI: 10.1037/a0014510 (2009).

29. Marwan, N. \& Webber, C. L. Mathematical and Computational Foundations of Recurrence Quantifications. In Webber, C. L. \& Marwan, N. (eds.) Recurrence Quantification Analysis, 3-43, DOI: 10.1007/978-3-319-07155-8_1 (Springer International Publishing, Cham, 2015). Series Title: Understanding Complex Systems.

30. Schiavio, A., Maes, P.-J. \& van der Schyff, D. The dynamics of musical participation. Music. Sci. 1029864920988319 (2021).

31. Clark, A. Natural-Born Cyborgs: Minds, Technologies, and the Future of Human Intelligence (Oxford University Press, 2003).

32. Theiner, G., Allen, C. \& Goldstone, R. L. Recognizing group cognition. Special Issue on Ext. Mind 11, 378-395, DOI: 10.1016/j.cogsys.2010.07.002 (2010).

33. Kirchhoff, M. D. \& Kiverstein, J. Attuning to the World: The Diachronic Constitution of the Extended Conscious Mind. Front. Psychol. 11, 1966, DOI: 10.3389/fpsyg.2020.01966 (2020).

34. Spivey, M. Who You Are: the Science of Connectedness (MIT Press, 2020).

35. O'Regan, J. K. \& Noë, A. A sensorimotor account of vision and visual consciousness. Behav. Brain Sci. 24, 939-973, DOI: $10.1017 / \mathrm{S} 0140525 X 01000115$ (2001).

36. Goupil, L., Saint-Germier, P., Rouvier, G., Schwarz, D. \& Canonne, C. Musical coordination in a large group without plans nor leaders. Sci. Reports 10, 1-14 (2020).

37. Tunçgenç, B., Travers, E. \& Fairhurst, M. T. Leadership and tempo perturbation affect coordination in medium-sized groups. Sci. reports 11, 1-11 (2021).

38. Demos, A. P., Chaffin, R., Begosh, K. T., Daniels, J. R. \& Marsh, K. L. Rocking to the beat: Effects of music and partner's movements on spontaneous interpersonal coordination. J. Exp. Psychol. Gen. 141, 49-53, DOI: 10.1037/a0023843 (2012).

39. von Zimmermann, J., Vicary, S., Sperling, M., Orgs, G. \& Richardson, D. C. The Choreography of Group Affiliation. Top. Cogn. Sci. 10, 80-94, DOI: 10.1111/tops.12320 (2018).

40. Vicary, S., Sperling, M., von Zimmermann, J., Richardson, D. C. \& Orgs, G. Joint action aesthetics. PLOS ONE 12, e0180101, DOI: 10.1371/journal.pone.0180101 (2017).

41. McEllin, L., Knoblich, G. \& Sebanz, N. Synchronicities that shape the perception of joint action. Sci. Reports 10, 15554, DOI: 10.1038/s41598-020-72729-6 (2020).

42. Wiltermuth, S. S. Synchronous activity boosts compliance with requests to aggress. J. Exp. Soc. Psychol. 48, 453-456, DOI: 10.1016/j.jesp.2011.10.007 (2012).

43. Allen, M. et al. Raincloud plots: a multi-platform tool for robust data visualization [version 2; peer review: 2 approved]. Wellcome Open Res 4, DOI: 10.12688/wellcomeopenres.15191.1. (2021).

44. Wickham, H. ggplot2: Elegant Graphics for Data Analysis (Springer, 2016), 2 edn.

45. Bates, D. Fitting Linear Mixed-Effects Models Using lme4. J. Stat. Softw. DOI: 10.18637/jss.v067.i01 (2015).

46. Demos, A. P., Chaffin, R. \& Logan, T. Musicians body sway embodies musical structure and expression: A recurrence-based approach. Music. Sci. 22, 244-263, DOI: 10.1177/1029864916685928 (2017).

\section{Acknowledgements}

The authors wish to thank Byron Pillow, M.M. (National Music Museum, University of South Dakota) for providing technical assistance and music theory consultation, and Dr Paul Garza, D.M.A. (University of Houston) for music theory consultation.

This material is based upon work supported by the National Science Foundation under Grant No. DGE-1633722. Any opinions, findings, and conclusions or recommendations expressed in this material are those of the author(s) and do not necessarily reflect the views of the National Science Foundation. 


\section{Author contributions statement}

S.P. devised the project, the main conceptual ideas, and led writing the manuscript. M.R. performed data cleaning and preparation. S.P. and M.R. performed the nonlinear analysis, S.P performed statistical analysis. R.B. and M.S. verified the analytical methods and supervised the findings of this work. All authors discussed the results and contributed to the final manuscript. 